\title{
The provision of neuro-oncology and glioma neurosurgery during the SARS-CoV-2 pandemic: a single national tertiary centre experience
}

\author{
Michael Amoo ${ }^{1,2}$ (1) $\cdot$ Jack Horan $^{1} \cdot$ Brian Gilmartin $^{1} \cdot$ Deirdre Nolan ${ }^{1} \cdot$ Paula Corr $^{1} \cdot$ Stephen MacNally ${ }^{1}$. \\ Mohammed Ben Husien ${ }^{1,2} \cdot$ Mohsen Javadpour ${ }^{1,2,3}$
}

Received: 18 September 2020 / Accepted: 1 November 2020 / Published online: 5 November 2020

(C) Royal Academy of Medicine in Ireland 2020

\begin{abstract}
Background The COVID-19 pandemic has resulted in a significant disruption in the provision of healthcare globally. The aim of this study was to assess the implications of the COVID-19 pandemic on the provision of neuro-oncology surgery and comparison with a similar 3-month period in 2019.

Methods Retrospective review of prospectively curated database of patients requiring neuro-oncology surgery at our tertiary referral centre between 1st March 2020 and 31st May 2020. We also analysed data for the same time period (1st March-31st May) in 2019 for comparison. Number and type of tumours operated on, postoperative morbidity and mortality, COVID-19related complications and delays in treatment were recorded.

Results During the 3-month periods studied in 2020 and 2019, there were 127 and 139 admissions for neuro-oncological surgery, respectively. Sixty patients underwent surgery for gliomas during the 2020 period vs 56 in the 2019 period. We observed no increase in mean length of time from referral to inter-hospital transfer (mean of $76 \mathrm{~h}$ in $2020 \mathrm{vs} 93 \mathrm{~h}$ in $2019(p=0.10)$ ) or in mean length of time from admission to surgery in the acute admissions (2.39 days in 2020 vs 2.89 days in 2019). The postoperative 30 -day morbidity and mortality rates were lower in $2020 ; 8.7 \%(n=11)$ compared with $10.1 \%(n=14)$ in 2019 . There was one COVID-19-related death which occurred in a patient with B cell lymphoma with negative preoperative COVID-19 test.

Conclusion The provision of neuro-oncological surgery can be safely continued during respiratory illness epidemic or pandemic if a rigorous testing and staffing framework is implemented.
\end{abstract}

Keywords COVID-19 $\cdot$ Glioblastoma $\cdot$ Glioma $\cdot$ Neuro-oncology $\cdot$ Neurosurgery

\begin{tabular}{llll} 
Abbreviations & & CSF & Cerebrospinal fluid \\
Abx & Antibiotics & DNET & Dysembryoplastic neuroepithelial tumour \\
CN & Cranial nerve & EVD & External ventricular drain \\
CNS & Central nervous system & GBM & Glioblastoma multiforme \\
COVID-19 Coronavirus disease 2019 & GI & Gastrointestinal \\
& GTR & Gross total resection \\
& HB & House-Brackmann \\
\hline $\begin{array}{l}\text { Michael Amoo } \\
\text { michaelamoo@ rcsi.ie }\end{array}$ & HGG & High-grade glioma \\
& ICP & Intracranial pressure \\
National Centre for Neurosurgery, Beaumont Hospital, & ICU & Intensive care unit \\
Dublin, Ireland & KPI & Key performance index \\
2 Royal College of Surgeons in Ireland, Dublin, Ireland & LOHS & Length of hospital stay \\
3 & MDM & Multidisciplinary meeting \\
Department of Academic Neurology, Trinity College Dublin, & MRI & Magnetic resonance imaging \\
Dublin, Ireland & NTR & Near total resection
\end{tabular}




$\begin{array}{ll}\text { PAPR } & \text { Powered air-purifying respirator } \\ \text { P-LOHS } & \begin{array}{l}\text { Postoperative length of hospital stay } \\ \text { Personal protective equipment }\end{array} \\ \text { PPE } & \begin{array}{l}\text { Speech and language therapy } \\ \text { SALT }\end{array} \\ \text { SARS-CoV-2 } & \begin{array}{l}\text { Severe acute respiratory syndrome } \\ \text { coronavirus 2 }\end{array} \\ \text { Sim } & \text { Simpson's grade } \\ \text { SMA } & \begin{array}{l}\text { Supplemental motor area } \\ \text { STR }\end{array} \\ \text { Subtotal resection } \\ \text { TSS } & \text { Transsphenoidal surgery } \\ \text { WHO } & \text { World Health Organization }\end{array}$

\section{Introduction}

The coronavirus disease 2019 (COVID-19) pandemic has resulted in a significant disruption in the provision of healthcare globally. The primary burden of the pandemic has been clearly evident through the mortality, and pressure on hospital resources, directly related to the disease. However, the pandemic has also had an indirect effect on non-COVID patients, as a result of shortage of resources, cancellation and postponement of scheduled, non-urgent and semi-urgent patient care. In addition, social distancing measures necessary for controlling transmission of COVID-19 have led to limitations on number of attendees at neuro-oncology multidisciplinary meeting (MDMs) and in some cases temporary cessation of these meetings at our unit, although this was mitigated through the use of video-conferencing technology. Patients undergoing surgery are a vulnerable cohort for exposure to the severe acute respiratory syndrome coronavirus 2 (SARS-CoV-2). Furthermore, higher 30-day postoperative mortality rates and pulmonary complication rates have been reported in patients with peri-operative diagnosis of SARS-CoV-2 infection. In a recent international cohort study, there was a higher reported 30-day mortality rate in the cohort having surgery for cancer in comparison with patients with benign or obstetrics diagnosis $(\mathrm{OR}=1.55$ [1.01-2.39]) [1].

For malignant tumours of the central nervous system (CNS) as well as benign tumours which are causing significant mass effect, prompt neurosurgical treatment is paramount. During the COVID-19 pandemic, the benefits of such prompt surgical intervention need to be balanced against the risks to patients and staff posed by the Coronavirus itself. Several guidelines have been published for the management of cancer patients, including those with intracranial neoplasms, during the COVID-19 pandemic [2, 3]. These guidelines had to be drawn up at the beginning of the pandemic based on little available evidence and have been constantly modified during the course of the pandemic. As a result, different centres have adopted different approaches to provision of cancer surgery $[4,5]$. The aim of this study was to report the operative management of patients with CNS tumours at our centre during the peak COVID-19 pandemic period (MarchMay 2020) and to compare this with the same period in 2019.

\section{Methods and materials}

All neuro-oncological cases operated on at our centre since January 2012 have been prospectively entered into a dedicated database. This includes patient demographics, details of surgical procedures performed, extent of resection based on postoperative imaging, adjuvant treatment received, histology of the tumour and any postoperative neurological complications. From this prospective database, we chose 2 periods to study: 1st March to 31st May 2020 (the peak period for COVID-19 pandemic in Ireland) and 1st March to 31st May 2019. Patient records were also reviewed to document any delayed neurological complications and any non-neurological complications including COVID-19-related complications. There were several changes made to the peri-operative managements of all surgical patients our centre during the pandemic in 2020 . We have outlined these changes in Fig. 1.

As part of a dynamic quality improvement programme, there are a number of key performance indices (KPI) at our institution, including relevant KPIs for management of referrals. The KPI for inter-hospital transfers for neuro-oncology referrals is for admission to our unit from referring hospitals to be completed within 5 working days (Monday-Friday) or 7 days (including weekends), from the time of referral.

\section{Data analysis}

The data presented is mainly descriptive; hence analyses have been presented as proportions and percentages. Paired $t$ test analyses were performed to compare the means of waiting times and length of hospital stay between two cohorts.

\section{Results}

The total number of admissions for neuro-oncology surgery in March to May 2020 and March to May 2019 were 127 and 139 , respectively (Tables 1 and 2). Glial tumours represented $47 \%(n=60)$ of operated tumours in 2020 vs $40 \%(n=56)$ in 2019. Metastases represented $23 \%(n=29)$ of tumours in 2020 vs $13 \%(n=18)$ in 2019. Meningiomas accounted for $10 \%(n=13)$ of tumours in 2020 vs $14 \%(n=20)$ in 2019 . No transsphenoidal pituitary surgery was performed during the period of interest in 2020 vs 19 pituitary tumours operated on during the period of interest in 2019. The extent of tumour resection for certain tumour categories during 2020 and 2019 is presented in Table 3 . 


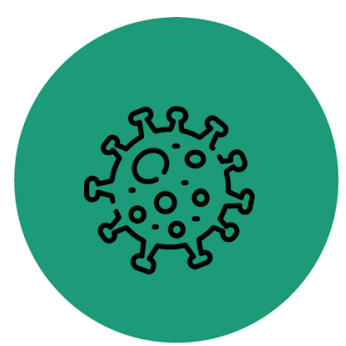

Pre-operative SARS-CoV2 testing

- Negative result confirmed from referring hospital (if time permitting)

- Nursed in isolation on arrival until repeat test at our unit

- Surgery within 48 hours of negative result

- Elective: Outpatient test up to 48 hours before proposed surgery date

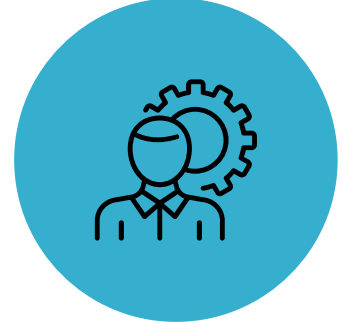

Theatre staff PPE

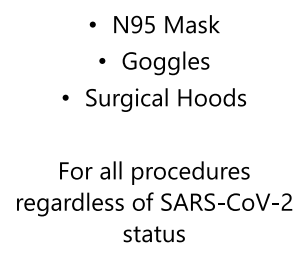

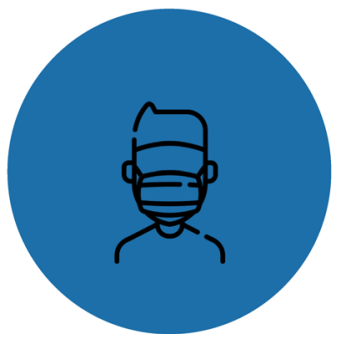

Intubation and Extubations

- Operating room (OR) vacated by non-anaesthesiology staff

- Remaining staff re-enter OR 10-15 minutes after intubation

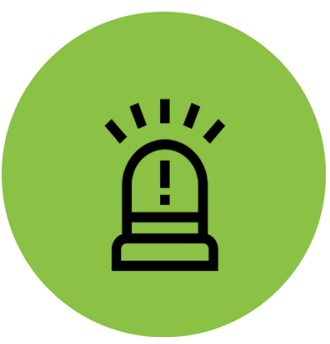

Emergency Surgery

- Operated on without testing

Fig. 1 Changes in peri-operative care of patients requiring emergency or scheduled neurosurgery

\section{Inter-hospital transfers}

There were 95 acute admissions due to CNS neoplasms during the 3-month period of interest in 2020, in comparison with 80 acute admissions in the 2019 period. Patient demographics, histological diagnoses, time intervals between referral and inter-hospital transfer as well as between transfer and surgery are presented in Table 1.

In $2020,88(92.6 \%)$ of the acute admissions were through inter-hospital transfers, which was similar to the 2019 figure of 75 (93.75\%). In comparison to 2019, there was less delay in inter-hospital transfer of these patients in 2020 (mean waiting time of $76 \mathrm{~h}$ in $2020 \mathrm{vs} 93 \mathrm{~h}$ in 2019 $(p=0.10)$ ), with only $4.4 \%(n=4)$ of patients breaching our key performance index (KPI), in comparison to $9.6 \%(n=7)$ in 2019 , for time taken for inter-hospital transfer of neuro-oncology patients. After weekend days were accounted for, the mean waiting time from admission to operation was less in 2020 (mean $=2.39$ days $\mathrm{SD}=1.87$ ) compared with the 2019 period $($ mean $=2.89$ days, $\mathrm{SD}=$ 2.06), $\mathrm{t}(172)=1.65, p=.0499$ (Table 1).

The mean overall length of hospital stay (LOHS) was shorter in 2020 (9.3 days) compared with 2019 (11.4 days); however this did not reach statistical significance $(p=0.054)$. There was no difference between the mean postoperative length of hospital stay (P-LOHS) between the 2 years $(6.1$ and 7.5 days for 2020 and 2019 respectively). In 2020, $53.7 \%(n=51)$ of patients were discharged home from our institution, and the rest required ongoing in-patient rehabilitation. In 2019, 56.3\% $(n=45)$ were discharged home. A $6.3 \%$ $(n=6) 30$-day morbidity rate was recorded in 2020, including one surgical site infection, in comparison to $8.75 \%(n=7) 30$ day morbidity in 2019, which also included one surgical site infection resulting in sepsis. Table 4 contains a summary of
Table 1 Demographics and comparison of acute (urgent) neurosurgical oncology cases between 2019 and 2020

\begin{tabular}{|c|c|c|}
\hline Urgent admissions & 2020 & 2019 \\
\hline & $n=95$ & $n=80$ \\
\hline \multicolumn{3}{|l|}{ Gender } \\
\hline Male & $48(50.5 \%)$ & $36(45 \%)$ \\
\hline Female & $47(45.5 \%)$ & $44(55 \%)$ \\
\hline Mean Age (years) & 55 & 58.4 \\
\hline Inter-hospital transfers & $88(92.6 \%)$ & $75(93.8 \%)$ \\
\hline Mean Transfer waiting time (hours) & 76 & 93 \\
\hline \multicolumn{3}{|l|}{ Met KPI for transfer time } \\
\hline Yes & $91(95.8 \%)$ & $73(91.3 \%)$ \\
\hline $\begin{array}{l}\text { Mean time from admission to } \\
\text { surgery (days) }\end{array}$ & 2.39 & 2.89 \\
\hline Glial tumours & $50(52.6 \%)$ & $39(48.75 \%)$ \\
\hline Pilocytic astrocytoma & - & $3(7.7 \%)$ \\
\hline Astrocytoma, WHO 2 & $3(6 \%)$ & $2(5.13 \%)$ \\
\hline Oligodendroglioma, WHO 2 & $3(6 \%)$ & - \\
\hline Astrocytoma, WHO 3 & $1(2 \%)$ & $1(2.56 \%)$ \\
\hline Astroblastoma & - & $1(2.56 \%)$ \\
\hline Gliosarcoma & - & $1(2.56 \%)$ \\
\hline GBM & $43(86 \%)$ & $31(79.49 \%)$ \\
\hline Non-glial tumours & $45(47.4 \%)$ & $41(51.25 \%)$ \\
\hline Pituitary tumours & - & $10(24.39 \%)$ \\
\hline Metastatic tumours & $25(55.56 \%)$ & $15(36.59 \%)$ \\
\hline Others* & $20(44.44 \%)$ & $16(39.02 \%)$ \\
\hline
\end{tabular}

KPI key performance index, WHO World Health Organization

*Includes neurocytoma, lymphoma, germinoma, ependymoma, haemangioblastoma, medulloblastoma, meningioma, schwannoma and solitary fibrous tumours 
Table 2 Demographics and comparison of elective neurosurgical oncology cases between 2019 and 2020

\begin{tabular}{lll}
\hline Elective admissions & 2020 & 2019 \\
\hline & $n=32$ & $n=59$ \\
Gender & $15(46.9 \%)$ & $31(52.5 \%)$ \\
Male & $17(53.1 \%)$ & $28(47.5 \%)$ \\
Female & 50.5 & 46 \\
Mean Age (years) & $2.2(1.7-2.9)$ & $1.2(1.07-1.41)$ \\
Mean time from admission & $\mathbf{1 0}(\mathbf{3 1 . 2 5 \%})$ & $\mathbf{1 7}(\mathbf{2 8 . 8 \% )}$ \\
to surgery (days) & - & $1(5.9 \%)$ \\
Glial tumours & $2(20 \%)$ & $6(35.3 \%)$ \\
Pilocytic astrocytoma & $1(10 \%)$ & - \\
Astrocytoma, WHO 2 & $4(40 \%)$ & $1(5.9 \%)$ \\
Oligodendroglioma, WHO 2 & - & $1(5.9 \%)$ \\
Astrocytoma, WHO 3 & $3(30 \%)$ & $8(47 \%)$ \\
Astroblastoma & $\mathbf{2 2 ~ ( 6 8 . 7 5 \% )}$ & $\mathbf{4 2} \mathbf{( 7 1 . 2 \% )}$ \\
GBM & 0 & $9(21.43 \%)$ \\
Non-glial tumours & $4(18.18 \%)$ & $3(7.14 \%)$ \\
Pituitary tumours & $18(81.82 \%)$ & $30(71.43 \%)$ \\
Metastatic tumours & & \\
Others* & &
\end{tabular}

DNET dysembryoplastic neuroepithelial tumour, GBM glioblastoma multiforme,

KPI key performance index, WHO World Health Organization

*Includes colloid cyst, dysembryoplastic neuroepithelial tumour, lymphoma, germioma, ependymoma, myxopapillary Ependymoma, haemangioblastoma, meningioma, schwannoma, endo-dermal cyst and chondrosarcoma

recorded morbidities. There was one postoperative mortality each in 2019 and 2020, and both were as a consequence of rapid tumour progression.

Only 1 patient tested positive for COVID-19 preoperatively. This patient underwent delayed surgery. This patient, with a background history of high-grade B cell lymphoma, was referred from another institution following an incidental finding of a right parietal lesion identified on surveillance MRI. The patient had a negative test for SARS-CoV-2 at the referring hospital $24 \mathrm{~h}$ prior to inter-hospital transfer. Although the patient was asymptomatic, a repeat test was performed $24 \mathrm{~h}$ before the proposed surgery, according to protocols at our unit. This repeat yielded a positive result. The surgery was deferred, and the patient was discharged home, advised to self-isolate, and was subsequently re-admitted from home after 21 days. Repeat testing was negative. A craniotomy and gross total resection (GTR) was performed with all operating room staff in full PPE.

\section{Elective admissions}

There were 32 elective admission for CNS tumours in 2020, in comparison with 59 in 2019. Patient demographics, histological diagnoses, time interval between admission and surgery are
Table 3 Extents of resection (With intra-operative and postoperative imaging correlations)

\begin{tabular}{lll}
\hline & 2020 & 2019 \\
\hline $\begin{array}{l}\text { High-grade glioma } \\
\text { Biopsy }\end{array}$ & $19(37.25 \%)$ & $16(39 \%)$ \\
STR & $17(33.3 \%)$ & $15(36.6 \%)$ \\
NTR & $7(13.76 \%)$ & $2(4.9 \%)$ \\
GTR & $8(15.69 \%)$ & $8(19.5 \%)$ \\
Meningioma & & \\
Sim 1 & $2(15.4 \%)$ & $1(5 \%)$ \\
Sim 2 & $9(69.2 \%)$ & $10(50 \%)$ \\
Sim 3 & $1(7.7 \%)$ & $3(15 \%)$ \\
Sim 4 & $1(7.7 \%)$ & $5(25 \%)$ \\
TSS (STR) & - & $1(5 \%)$ \\
Others & & $10(13.7 \%)$ \\
Biopsy & $14(19.2 \%)$ & $28(38.4 \%)$ \\
STR & $8(11 \%)$ & $3(4.1 \%)$ \\
NTR & $12(16.4 \%)$ & $32(43.8 \%)$ \\
GTR & $39(53.4 \%)$ & \\
\hline
\end{tabular}

GTR gross total resection, NTR near total resection, STR subtotal resection, Sim Simpson's grade, TSS transsphenoidal surgery

presented in Table 2. After weekend days are accounted for, the mean waiting time from admission to operation was longer by 1 day in 2020 (mean $=2.2$ days $(1.7-2.9$ days) vs mean $(2019)=1.2$ days $(1.07-1.41$ days $))($ Table 2$)$. The mean overall length of hospital stay was shorter in 2020 (7.3 days) compared with 2019 (10.6 days); however there was no statistical significance to this observation $(p=0.13)$. All patients were discharged home, with the exception of one patient who was discharged to a rehabilitation facility in 2020. A $9 \%(n=3) 30$ day morbidity rate was recorded in 2020 , with no surgical site infections, in comparison to $10 \%(n=6) 30$-day morbidity in 2019, including 2 surgical site-related infections (Table 4). There was one 30-day mortality recorded in 2020 . This was a patient who had a left frontal diffuse large B cell lymphoma, confirmed on biopsy. The patient had a negative preoperative test for the SARS-CoV-2 virus. The patient was discharged home on the 8th postoperative day, however, presented to another hospital on the 18th postoperative day with respiratory symptoms and was subsequently diagnosed with COVID-19. The patient subsequently died of COVID-19-related complications on the 30th postoperative day.

\section{High-grade glioma (HGG) subgroup}

Gliomas accounted for $47.24 \%(n=60)$ of neuro-oncological tumours treated during the time period of interest in 2020, of which $85 \%(n=51)$ were high-grade glial tumours (Astrocytoma, WHO 3 and 4). During the same time period 
Table 4 Summary of postoperative morbidities

\begin{tabular}{|c|c|c|c|}
\hline & Diagnosis & Morbidity & Management \\
\hline \multicolumn{4}{|l|}{2020} \\
\hline \multicolumn{4}{|c|}{ Urgent cases } \\
\hline 1 & GBM & Pulmonary embolism & Anticoagulation \\
\hline 2 & Central neurocytoma & Hydrocephalus & EVD \\
\hline 3 & Oligodendroglioma, WHO 2 & Dysphasia & SALT \\
\hline 4 & Metastatic adenocarcinoma (Breast Primary) & Symptomatic haematoma & Conservative \\
\hline 5 & Vestibular Schwannoma & HB-3 Facial weakness & Supportive \\
\hline 6 & Oligodendroglioma, WHO 2 & Surgical site infection (Sepsis) & Abx, EVD, Craniectomy \\
\hline \multicolumn{4}{|c|}{ Elective cases } \\
\hline 1 & Astrocytoma, WHO 3 & SMA syndrome & Physiotherapy \\
\hline 2 & Astrocytoma, WHO 2 & CSF leak & Wound re-suturing \\
\hline 3 & Vestibular Schwannoma & HB-1 facial weakness & Supportive \\
\hline \multicolumn{4}{|l|}{2019} \\
\hline \multicolumn{4}{|c|}{ Urgent cases } \\
\hline 1 & Metastatic adenocarcinoma (GI primary) & Pneumonia & Antibiotics \\
\hline 2 & GBM & Severe cerebral oedema & Surgical decompression \\
\hline 3 & Pituitary Adenoma & Epistaxis & Conservative \\
\hline 4 & Haemangioblastoma & Pseudomeningocoele & Conservative \\
\hline 5 & Meningioma WHO 1 & Subdural haematoma & Craniectomy \\
\hline 6 & GBM & Intracranial haemorrhage & Craniectomy \\
\hline 7 & Pituitary Adenoma & Diabetes Insipidus & Desmopressin \\
\hline \multicolumn{4}{|c|}{ Elective cases } \\
\hline 1 & Endo-dermal Cyst & Hearing loss & Supportive \\
\hline 2 & Meningioma, WHO 1 & CSF Rhinorrhoea & Lumbar drain \\
\hline 3 & GBM & Surgical site infection & Surgery, Abx \\
\hline 4 & Ependymoma, WHO 2 & Spinal cord Herniation & Surgery \\
\hline 5 & Meningioma WHO 1 & Surgical site infection, Meningitis & Abx \\
\hline 6 & Meningioma WHO 1 & Hemiparesis, Airway obstruction, CN VI and VII palsy & Supportive \\
\hline
\end{tabular}

$A b x$ antibiotics, $C N$ cranial nerve, $C S F$ cerebrospinal fluid, $E V D$ external ventricular drain,

GI gastrointestinal, GTR glioblastoma multiforme, $H B$ House-Brackmann,

SALT speech and language therapy, SMA supplementary motor Area, WHO World Health Organization

in $2019,40.3 \%(n=56)$ of patients had glioma, of which $78.6 \%(n=44)$ were high grade (including Astrocytoma WHO 3 and 4, Astroblastoma and Gliosarcoma) (Tables 1 and 2). In 2020, $16.7 \%(n=10)$ of patients undergoing surgery for glioma were admitted electively from home vs $30.4 \%$ $(n=17)$ in 2019.

For HGG patients transferred from other hospitals, there was no significant difference in the mean time to transfer completion for patients with HGG between 2020 and 2019 (mean times of 86.3 and $87.6 \mathrm{~h}$, respectively). The overall mean time from admission to surgery for patients with HGG was 2.3 days in 2020, in comparison to 2.8 days in 2019 following correction for weekend hospital stays. There was a statistically significant difference in the overall mean length of hospital stay between the 2 years ( 7.7 days in 2020 vs 10.7 days in 2019, $p=0.03$ ); however, there was no difference in the length of postoperative hospital stay (4.4 days in 2020 vs 5.6 days in 2019, $p=0.15$ ). Fifty-two percent of HGG patients admitted urgently (non-elective) were discharged home postoperatively in 2020 vs $50 \%$ in 2019, and the rest were repatriated back to their referring hospitals for continued in-patient rehabilitation. The 30-day postoperative morbidity rate following HGG surgery in 2020 was $3.9 \%(n=2$, one patient had a pulmonary embolism and the other developed a supplemental motor area (SMA) syndrome), with no surgical site infections (Table 4), and 1 patient died from complications of their tumour progression postoperatively. The 30day post -operative morbidity for the same period in 2019 was $7 \%(n=3)$ overall, including 1 surgical site infection. One patient developed severe cerebral oedema 11 days after biopsy of a right frontal GBM, requiring emergency decompression and debulking of the tumour. The third patient had a large 
haematoma adjacent to the surgical cavity a few hours following debulking for a right temporal GBM. The patient subsequently required a decompressive craniectomy and a prolonged hospital stay before transfer back to the referring hospital for rehabilitation. There was one mortality in 2019 as a result of GBM progression.

\section{Discussion}

Bernhardt et al. presented a pragmatic consensus-based framework for prioritization and decision-making in the management of patients with high-grade glioma in the setting of potential severe strains on neurosurgical resources during the COVID-19 pandemic. These included prioritizing patients with neurological deficits, altering surgical intents to decompression rather than gross total resection (GTR) and even commencing radiation and chemotherapy in elderly patients without tissue diagnosis [5]. These recommendations were appropriately made to ensure protection of patients and healthcare providers against SARS-CoV-2 infection and also to provide the best possible care for patients in the face of stretched healthcare resources during the pandemic. However, it is important that we modify our guidelines as we learn more about the pandemic.

In our centre, we implemented several rigorous changes to staffing and preoperative screening policies and prioritization of cases during the pandemic, as outlined in detail in the "Methods and materials" section. Our data presented in this paper demonstrate that comparable volumes of neurooncological surgery were carried out during the pandemic in comparison to the same period in 2019. In particular, patients with malignant brain tumours received standard care, with no compromise in the extent and proportions of radical (gross total and near total) resections during the pandemic (Table 3). This was achieved without any demonstrable increase in morbidity or mortality, although one patient with high-grade B cell lymphoma and negative preoperative COVID-19 test subsequently developed SARS-CoV-2 infection which resulted in his death 30 days after his neurosurgical procedure.

We have also demonstrated that neuro-oncological surgery for urgent cases can be performed during the pandemic within similar time frames achieved in non-pandemic times. In fact, in our patient cohort, the time interval between patient referral and inter-hospital transfer was shorter during the pandemic compared to a similar period during 2019. This is likely to be due to the reduction in demand for neurosurgical beds as a result of the significant decrease in non-oncological neurosurgery (e.g. degenerative spinal disease) during the pandemic. It should also be pointed out that although the numbers of patients undergoing surgery for malignant brain tumours were similar in 2020 and 2019, the same was not true for benign tumours. During the 3-month period of the pandemic included in this study, we only operated on benign tumours if they were associated with significant mass effect on the brain or other critical structures such as the optic apparatus. This was particularly noticeable with regard to transsphenoidal surgery for pituitary adenomas. During the 3-month period studied (March to May 2020), no transsphenoidal operations were performed due to initial reports suggesting a very high risk of SARS-CoV-2 infection in operating room staff associated with transnasal procedures. From June 2020, we have commenced transsphenoidal surgery with all staff in the operating room wearing powered air-purifying respirator (PAPR). Further studies are required to quantify the risk of SARS$\mathrm{CoV}-2$ infection during transnasal procedures.

During the peak of the pandemic, there was only 1 patient who tested positive preoperatively for Coronavirus at our unit following a prior negative test at the referring hospital. This patient had no COVID-19 symptoms. They were discharged home for self-isolation and re-admitted electively for surgery after 14 days of isolation and a negative test. There were no cases of nosocomial COVID-19 infections reported in our surgical staff.

\section{Conclusion}

The provision of neuro-oncological surgery can be safely continued during respiratory illness epidemic or pandemic if a rigorous testing and staffing framework is implemented.

Funding No funding was received for this research.

\section{Compliance with ethical standards}

Conflict of interest All authors certify that they have no affiliations with or involvement in any organization or entity with any financial interest (such as honoraria; educational grants; participation in speakers' bureaus; membership, employment, consultancies, stock ownership or other equity interest; and expert testimony or patent-licencing arrangements) or nonfinancial interest (such as personal or professional relationships, affiliations, knowledge or beliefs) in the subject matter or materials discussed in this manuscript.

Ethical approval All procedures performed in studies involving human participants were in accordance with the ethical standards of the institutional and/or national research committee and with the 1964 Helsinki declaration and its later amendments or comparable ethical standards. For this type of study formal consent is not required.

\section{References}

1. COVIDSurg Collaborative (2020) Mortality and pulmonary complications in patients undergoing surgery with perioperative SARSCoV-2 infection: An international cohort study. Lancet (London, 
England) 396(10243):27-38.https://doi.org/10.1016/s01406736(20)31182-x

2. National Health Service (NHS) (2020) Specialty guides for patient management during the coronavirus pandemic - Clinical guide for the management of cancer patients during the coronavirus pandemic.https://www.uhb.nhs.uk/coronavirus-staff/downloads/ pdf/CoronavirusCancerManagement.pdf. Accessed 4 Sept 2020

3. Society of British Neurological Surgeons (2020) Adult Neurooncology service provision during COVID-19 outbreak. https:// www.sbns.org.uk/index.php/policies-and-publications/covid/. Accessed 4 Sept 2020
4. COVIDSurg Collaborative (2020) Global guidance for surgical care during the COVID-19 pandemic. Br J Surg. https://doi.org/10.1002/ bjs. 11646

5. Bernhardt D, Wick W, Weiss SE et al (2020) Neuro-oncology management during the COVID-19 pandemic with a focus on WHO grade III and IV Gliomas. Neuro-Oncology 22(7):928-935. https:// doi.org/10.1093/neuonc/noaa113

Publisher's note Springer Nature remains neutral with regard to jurisdictional claims in published maps and institutional affiliations. 